ARTICLE

\title{
Silicon and oxygen synergistic effects for the discovery of new high-performance nonfullerene acceptors
}

Ying Qin ${ }^{1,6}$, Hui Chen², ${ }^{2}$ Jia Yao ${ }^{3}$, Yue Zhou' ${ }^{1}$ Yongjoon Cho ${ }^{4}$, Yulin Zhu², Beibei Qiu ${ }^{5}$, Cheng-Wei Ju (D) 1, Zhi-Guo Zhang ${ }^{3 凶}$, Feng He (iD ${ }^{2 凶}$, Changduk Yang $\mathbb{D}^{4}$, Yongfang $\mathrm{Li}^{5} \&$ Dongbing Zhao (D) ${ }^{1 凶}$

In organic electronics, an aromatic fused ring is a basic unit that provides $\pi$-electrons to construct semiconductors and governs the device performance. The main challenge in developing new $\pi$-skeletons for tuning the material properties is the limitation of the available chemical approach. Herein, we successfully synthesize two pentacyclic siloxy-bridged $\pi$ conjugated isomers to investigate the synergistic effects of $\mathrm{Si}$ and $\mathrm{O}$ atoms on the geometric and electronic influence of $\pi$-units in organic electronics. Notably, the synthesis routes for both isomers possess several advantages over the previous approaches for delivering conventional aromatic fused-rings, such as environmentally benign tin-free synthesis and few synthetic steps. To explore their potential application as photovoltaic materials, two isomeric acceptor-donor-acceptor type acceptors based on these two isomers were developed, showing a decent device efficiency of $10 \%$, which indicates the great potential of this SiObridged ladder-type unit for the development of new high-performance semiconductor materials.

\footnotetext{
${ }^{1}$ State Key Laboratory and Institute of Elemento-Organic Chemistry, College of Chemistry, Nankai University, Tianjin 300071, China. ${ }^{2}$ Shenzhen Grubbs Institute and Department of Chemistry, Southern University of Science and Technology, Shenzhen 518055, China. ${ }^{3}$ State Key Laboratory of Chemical Resource Engineering, Beijing Advanced Innovation Center for Soft Matter Science and Engineering, Beijing University of Chemical Technology, Beijing 100029, China. ${ }^{4}$ Department of Energy Engineering, School of Energy and Chemical Engineering, Perovtronics Research Center, Low Dimensional Carbon Materials Center, Ulsan National Institute of Science and Technology (UNIST), Ulsan 44919, Republic of Korea. ${ }^{5}$ Beijing National Laboratory for Molecular Sciences, CAS Key Laboratory of Organic Solids, Institute of Chemistry, Chinese Academy of Sciences, Beijing 100190, China. ${ }^{6}$ These authors contributed

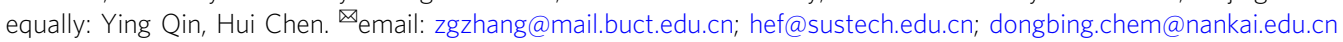


n comparison with its inorganic counterpart, such as classical silicon, organic electronics can promise unique features of solution processability, low weight, and flexibility ${ }^{1-9}$. These attractive advantages have inspired tremendous efforts in exploring the polycyclic aromatic hydrocarbons that can provide $\pi$-electrons to construct organic semiconductors, thus governing the device performance in a wide range of emerging technologies $^{10-15}$, such as organic light-emitting diodes (OLEDs), organic thin-film transistors (OFTs), and organic solar cells (OSCs). Although organic electronics have the highest market potential, their performances have not reached the practical requirement for broad commercial applications, mainly due to the suboptimal photophysical and electronic properties of these organic semiconductors. Fortunately, the properties of organic materials, including energy level, absorption, crystallinity, mobility, and electron affinity and stability in ambient air, can be flexibly adjusted by changing their chemical composition, such as the innovation of fused $\pi$-electron systems for obtaining highperformance polymeric and organic electronic materials ${ }^{16-19}$. In these bridged $\pi$-skeletons, the structural coplanarity and rigidity can reduce the conformational disorder, facilitate $\pi$-electron delocalization, and ultimately tune the photophysical and electronic properties.

In this context, the incorporation of silicon or oxygen atoms into the $\pi$-fused framework as bridging moieties has become a fruitful strategy to produce new $\pi$-materials possessing superb photophysical and electronic properties ${ }^{20-25}$, which are difficult to achieve in carbon-based $\pi$-units. The longer $\mathrm{C}-\mathrm{Si}$ bond length (C-Si vs. C-C: $\sim 1.87 \AA$ vs. $1.53 \AA$ ), higher electropositivity ( $\mathrm{Si}$ vs. C: 1.74 vs. 2.50), larger atomic radius (Si vs. C: $111 \mathrm{pm} v s .67$ $\mathrm{pm})$ and the particular interactions $\left(\sigma^{*}-\pi^{*}\right.$ conjugation) between the $\mathrm{Si}$ atom and the $\pi$-electron system often led to variation in energy levels, increased crystallinity, improved packing ability and higher charge mobility in comparison to those carbonbridged $\pi$-conjugated scaffolds (Fig. 1a) ${ }^{26-29}$. Indeed, diverse 5membered silicon-bridged $\pi$-systems, such as siloles, dibenzosiloles, dithienosiloles, and related compounds, have been synthesized and widely utilized in organic electronics (Fig. 1b) ${ }^{29-41}$. For example, Yang has shown that replacing carbon bridges with silicon bridges in cyclopentadithiophene-based polymers significantly improved the photovoltaic performance of polymer: PCBM-based solar cells ${ }^{42}$. On the other hand, recent studies revealed that the incorporation of electron-rich oxygen atoms into the bridge of ladder-type units would greatly improve the light-harvesting capability and the electron-donating capability of the unit, thus bringing unique characteristics to those new organic semiconductor materials to enhance the power conversion efficiency of organic photovoltaics ${ }^{43}$. Taking advantage of silicon and oxygen atom doping in the design of highperformance organic semiconductors, the development of 6membered silicon-oxygen-bridged ( $\mathrm{SiO}$-bridged) $\pi$-conjugated skeletons to investigate the synergistic effects of $\mathrm{Si}$ and $\mathrm{O}$ atoms in organic electronics is highly appealing and would significantly enhance the structural diversity of $\pi$-conjugated materials and improve the performance in a wide range of optoelectronic technologies. However, until now, investigations on 6-membered $\mathrm{SiO}$-bridged $\pi$-conjugated materials have been inherently limited by their narrow structural diversity, presumably mainly because of the challenges in their synthesis.

Herein, we designed two isomers of 6-memebered SiO-bridged ladder $\pi$-skeletons with different positions of silicon atoms, as shown in Fig. 1c. The silicon atom was connected to two thiophenes in SiO5T-5; in contrast, it was connected to the central benzene in SiO5T-10. This design aims to produce new donor units for the further development of high-performance organic/ polymeric semiconductors. Several aspects of these two skeletons are of interest. First, the thermal stability of siloxy-bridged ladder $\pi$-skeletons is expected to be higher because of the much larger average bond dissociation energy of the $\mathrm{Si}-\mathrm{O}$ bond. Moreover, according to density functional theory (DFT) calculations on the molecular orbital using Gaussian 09 at the B3LYP/6-31 G(d) level, the $\mathrm{Si}-\mathrm{O}$ bridge significantly alters the electronic structure of their $\mathrm{C}-\mathrm{O}$ analogs. As shown in Fig. 1d, the 6-memebered SiObridged structures significantly enhance $\pi$-system planarity $\left(10.9^{\circ}\right.$ vs. $0^{\circ} ; 8.5^{\circ}$ vs. $3.8^{\circ}$ ) compared with the CO-bridged analogs. The enhanced planar configuration would be beneficial for charge transport properties. Furthermore, the prediction of the energy levels by the DFT calculation showed that the SiO-bridged SiO5T-5 and SiO5T-10 possess larger band gaps with lower-lying HOMO energy levels $\left(E_{\mathrm{HOMO}}\right)$ than their carbon analogs. For the two siloxy-bridged $\pi$-skeletons of SiO5T-5 and SiO5T-10, the calculated $E_{\mathrm{LUMO}} / E_{\mathrm{HOMO}}$ of SiO5T-5 is $-1.52 /-5.07 \mathrm{eV}$, which is significantly lower than that of the isomer SiO5T-10 $\left(E_{\mathrm{LUMO}} /\right.$ $\left.E_{\mathrm{HOMO}}=-1.34 /-4.98 \mathrm{eV}\right)$, indicating the stronger interaction of the $\sigma^{*}$ orbital of the $\mathrm{Si}$ atom with the $\pi^{*}$ orbital of the $\pi$-skeleton in SiO5T-5. However, we may face some formidable challenges in our efforts to access the two siloxy-bridged ladder-type $\pi$-systems: (1) retro-Brook rearrangement may frequently occur if containing the siloxy-bridge in starting materials; (2) the instability of thiophenols is expected to lead to difficulty in the preparation of siloxy-bridged thiophenes. In this study, we addressed these problems in chemical synthesis and successfully accessed the two pentacyclic siloxy-bridged $\pi$-conjugated isomers SiO5Ts for the first time. Furthermore, we demonstrated that the incorporation of a $\mathrm{SiO}$ bridge into $\pi$-conjugated frameworks makes it possible to precisely tune the optoelectronic properties and molecular packing of $\pi$-systems, resulting in a decent device efficiency of $10 \%$ in polymer solar cells (PSCs) by the development of two novel isomeric siloxy-bridged A-D-A acceptors, which consist of our developed 6-membered laddered-SiO5T skeleton as the central donor unit and a dicyanomethylene derivative as the acceptor end group. This work is expected to enhance the discovery of high-performance materials for organic electronics using the 6-membered siloxy-bridged $\pi$ - skeleton as the central core.

\section{Results}

Skeleton synthesis. As mentioned above, the two ladder-type $\pi$ skeleton SiO5Ts have never been synthesized before. Herein, we tried to make a synthetic blueprint providing concise and rapid access to both isomers. In principle, the dibenzooxasiline moiety of the two ladder-type skeletons can be synthesized by $\mathrm{Pd}$ catalyzed $\mathrm{C}-\mathrm{H}$ bond arylation ${ }^{44}$, Rh-catalyzed transmetalation ${ }^{45}$ and $\mathrm{Rh}$-catalyzed intramolecular $\mathrm{C}-\mathrm{H}$ bond silylation ${ }^{46}$. However, both transmetalation and $\mathrm{C}-\mathrm{H}$ bond silylation involved the corresponding phenols as key intermediates. Considering the instability of thiophenol, we envisioned that Pd-catalyzed intramolecular arylation of the $\mathrm{C}-\mathrm{H}$ bond might be the only logical way to deliver the two siloxy-bridged ladder-type $\pi$-skeletons designed by us; even the competitive retro-Brook rearrangement can also occur during the $\mathrm{C}-\mathrm{H}$ activation step $^{47}$. We then carried out the synthesis of the two SiO5T isomers by intramolecular C $-\mathrm{H}$ bond arylation cyclization, as shown in Fig. 2. The lithium halogen exchange reaction of 3-bromothiophene 1 with ${ }^{n} \mathrm{BuLi}$ followed by treatment with dichlorodialkylsilane yielded chlorodialkyl(thiophen-yl)silane 2 , which further reacted with 2,5dibromohydroquinone 3 to deliver the corresponding bissilyl ether 4 , followed by subsequent $\mathrm{Pd}$-catalyzed $\mathrm{C}-\mathrm{H}$ bond arylation to give double-cyclized SiO-bridged SiO5T-5 (Fig. 2a). Following this success, we proceeded to synthesize the isomer of SiObridged SiO5T-5. After numerous attempts, we found that the 
a

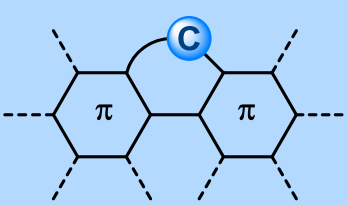

Fine-tuning the energy levels Improving the charge mobility and stability

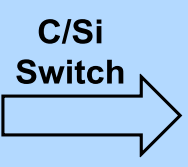

b Well-known silicon-bridged $\pi$-systems

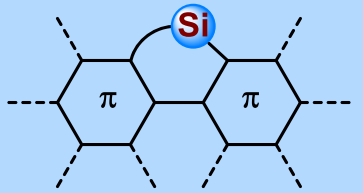

- Larger C-Si bond length

- Larger atomic radius

- Higher electropositivity

- $\sigma^{*}-\pi^{*}$ conjugation $\checkmark$ Increasing the crystallinity Streamlining synthesis
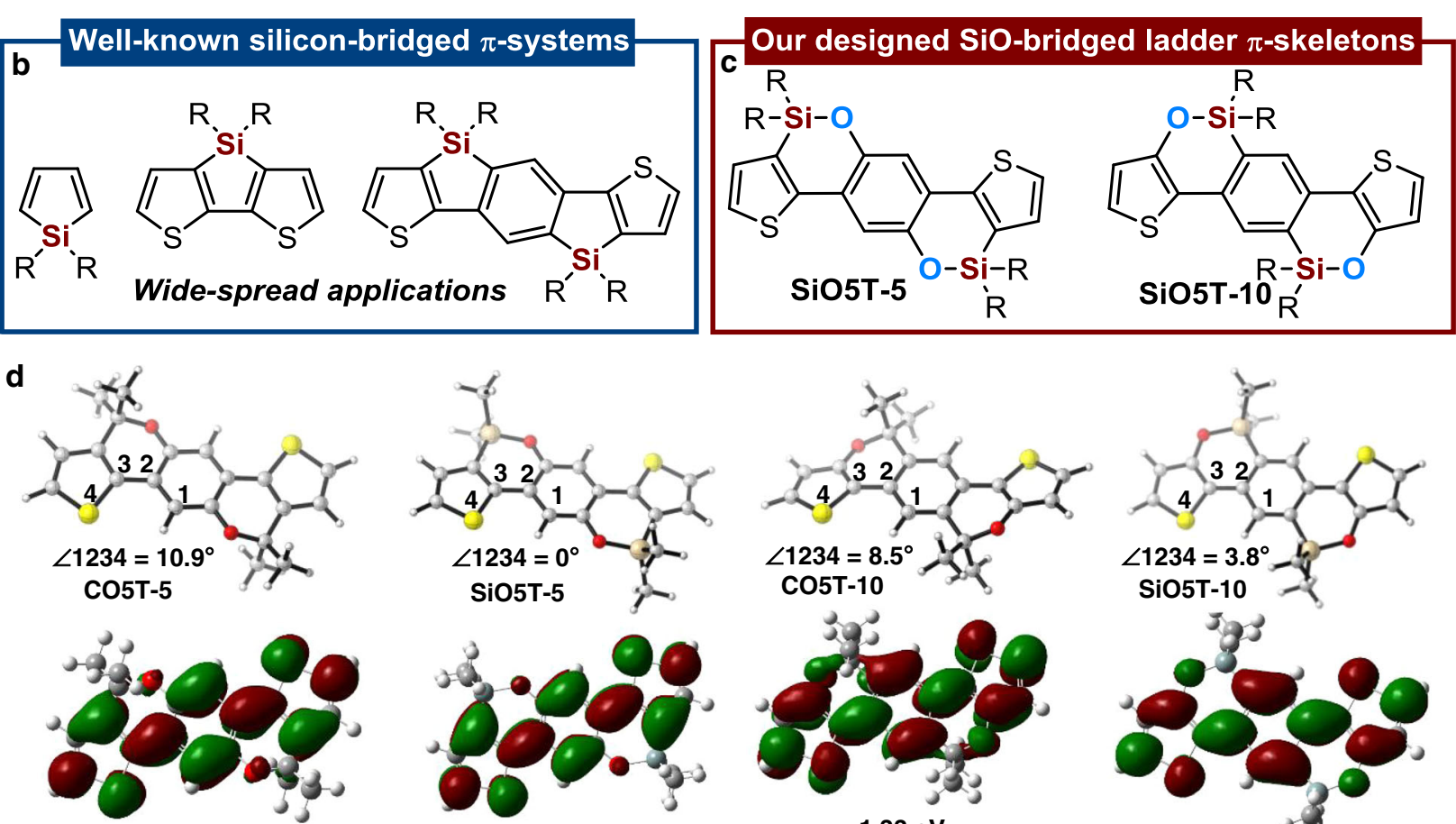

$-1.43 \mathrm{eV}$

$-1.52 \mathrm{eV}$
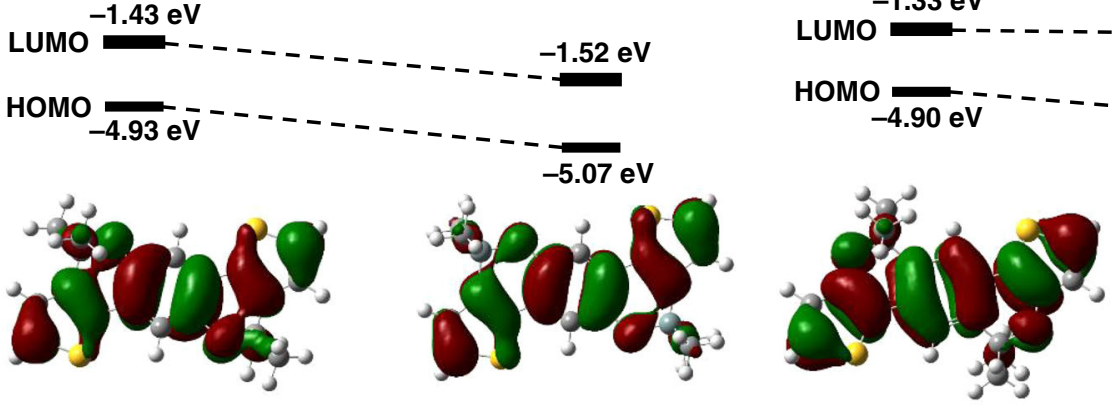

$-1.34 \mathrm{eVY}_{3}$
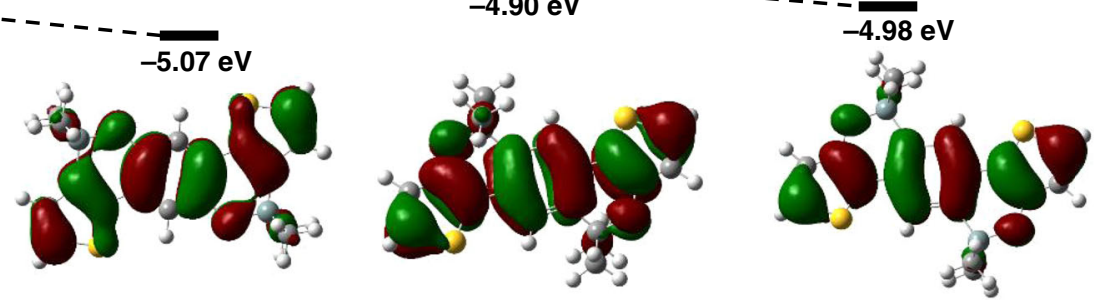

Fig. 1 Design and development of 6-membered SiO-bridged ladder-type units. a The advantage of incorporating silicon into $\pi$-systems. b Well-known silicon-bridged $\pi$-systems. c Our design on 6-membered SiO-bridged ladder-type units. $\mathbf{d}$ The molecular geometries and electron distributions of frontier orbitals of SiO5Ts and their carbon-analogs CO5Ts.

isomer SiO5T-10 can also be easily approached in a four-step synthesis from 1,4-dibromobenzene (Fig. 2b). The C2 and C5positions of the 1,4-dibromobenzene were lithiated by LDA and subsequently quenched with chlorodialkylhydrosilane, yielding bis-monohydrosilane 7 as a colorless oil. Further silane chlorination with TCCA and then reaction with 3-thiophenol in the presence of DMAP and imidazole in DCM afforded bissilyl ether 9 in 52\% yield. Finally, the isomer SiO5T-10 was obtained through a Pd-catalyzed intramolecular cyclization of aromatic $\mathrm{C}-\mathrm{H}$ bonds. In general, the synthetic routes for both SiO5T-5 and SiO5T-10 possess several advantages over the approaches for delivering the corresponding $\mathrm{C}$ or CO-bridged IDTs and CO5Ts, such as low cost, tin-free synthesis, and few synthetic steps.
Development of SiO-bridged acceptor materials. The development of new $n$-type small-molecule semiconductor materials as electron acceptors in bulk heterojunction (BHJ) organic photovoltaics (OPVs) has progressed rapidly and attracted worldwide attention in recent years because of the advantages of these materials over typical fullerene derivatives in terms of structural flexibility and absorbent tunability, thermal/photochemical stability, solubility/processability and synthetic costs ${ }^{48-52}$. Among the reported nonfullerene acceptors, acceptor-donor-acceptor (A-D-A) nonfullerene acceptors based on ladder-type $\pi$-systems seem to be the most successful to date due to their high electron mobility, suitable electronic energy levels, low bandgap, broad and strong absorpiton ${ }^{53-56}$. The further development of a novel ladder-type backbone is the core to construct new A-D-A 

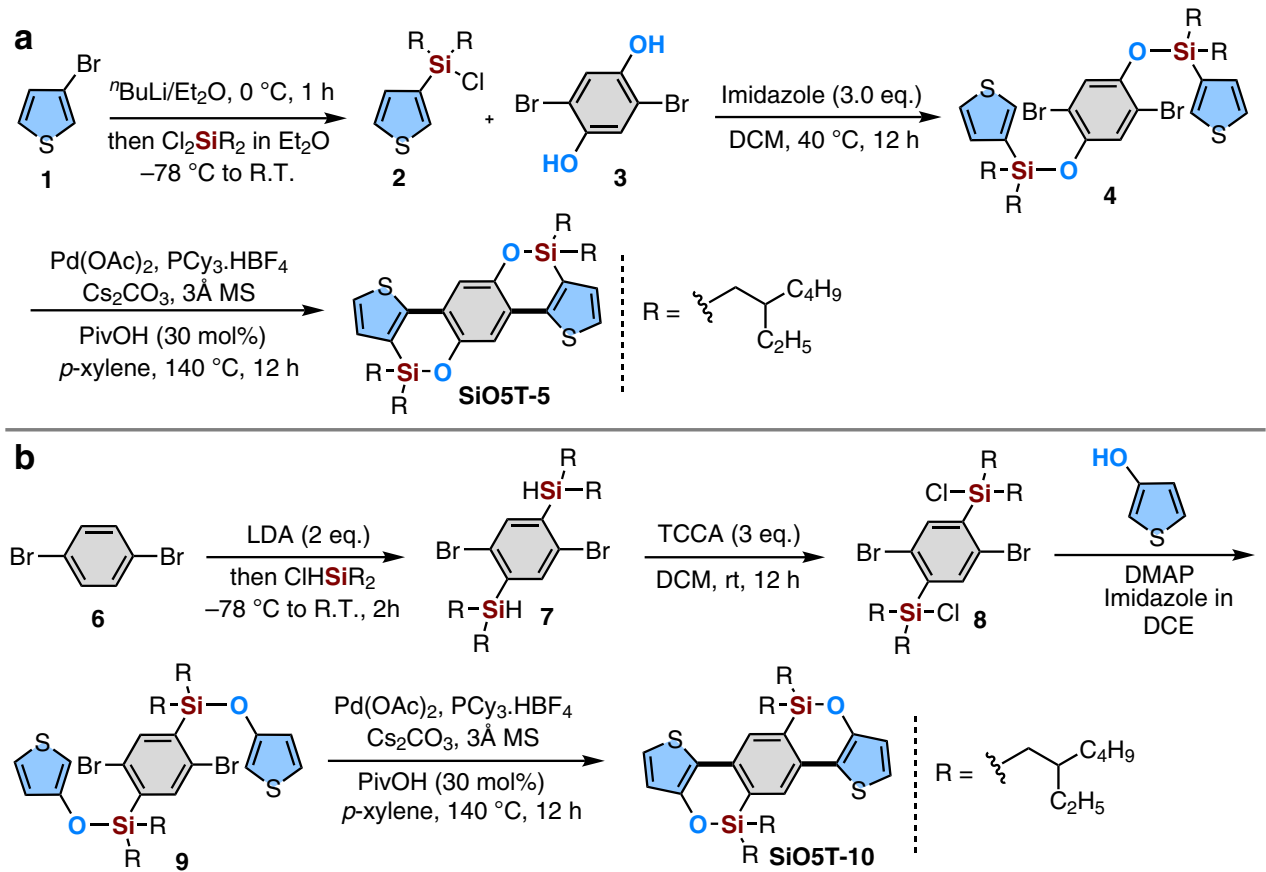

Fig. 2 Synthetic routes for the SiO-bridged ladder-type $\boldsymbol{\pi}$-skeletons. a The route for producing ladder-type $\pi$-skeleton SiO5T-5. $\mathbf{b}$ The route for producing ladder-type $\pi$-skeleton SiO5T-10.
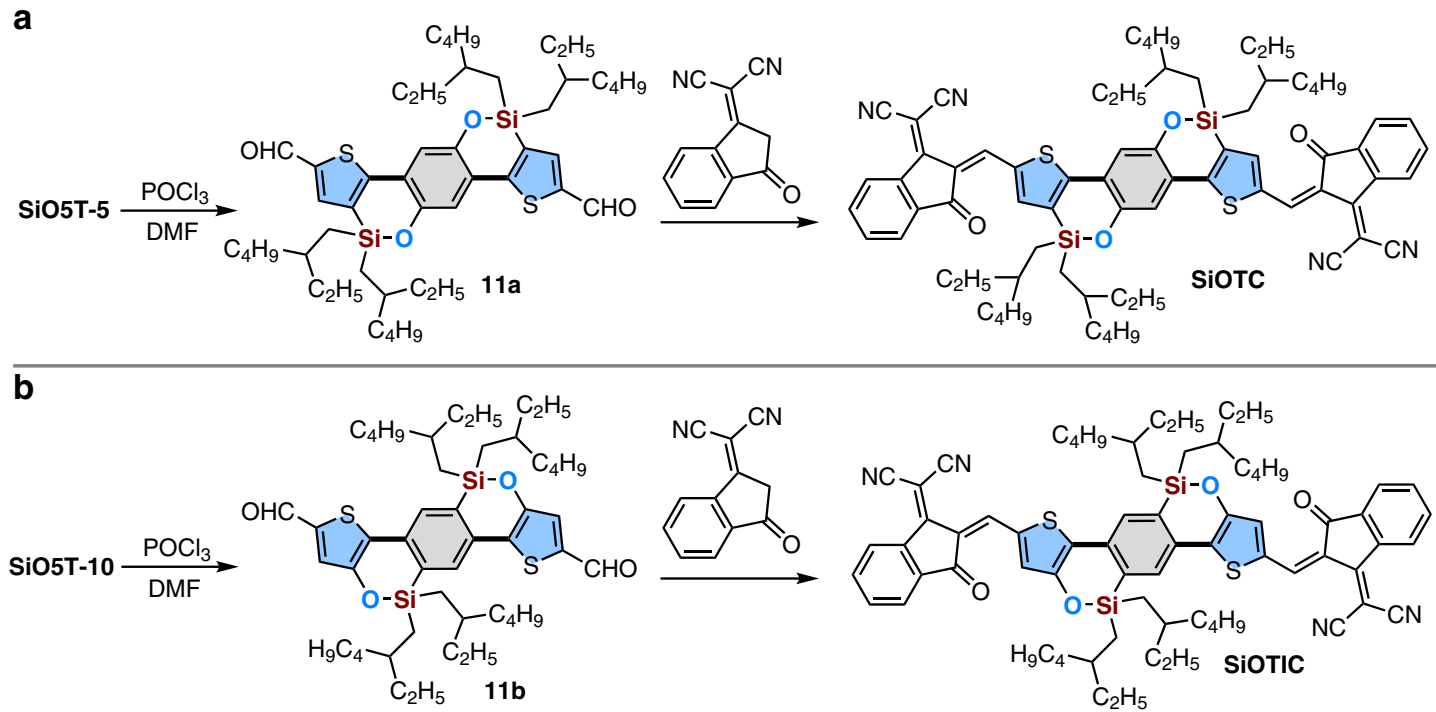

Fig. 3 Development of the two A-D-A nonfullerene acceptors based on ladder-type SiO-bridged $\boldsymbol{\pi}$-systems. a The synthetic route for the acceptor SiOTC. b The synthetic route for the acceptor SiOTIC.

nonfullerene acceptors for overcoming the issues in OPVs. Inspired by the good device performance obtained from those IDT-57-60 and CO5T-based ${ }^{61-65}$ ladder-type A-D-A nonfullerene acceptors, we speculated that the development of new A-D-A nonfullerene acceptors based on SiO-bridged ladder-type $\pi$ skeleton SiO5Ts can be a fruitful avenue for further improving the performance of nonfullerene PSCs because of silicon and oxygen synergistic effects. Herein, two isomers of A-D-A nonfullerene acceptors (SiOTC and SiOTIC) were designed and synthesized, as shown in Fig. 3, by bis-formylation of the C2position of thiophene units at SiO5T-5 or 10 to deliver the corresponding aldehyde, followed by condensation with the end group 2-(3-oxo-2,3-dihydroinden-1-ylidene)malononitrile (IC).

The optical properties of SiOTC and SiOTIC in dichloromethane (DCM) solution and in thin films were measured by
UV-vis absorption spectroscopy, as shown in Fig. 4a, b. The corresponding spectroscopic data are summarized in Table 1. These two isomers displayed very similar absorption profiles in solution with two peaks, which can be assigned as the (0-1) and (0-0) vibronic transitions, respectively (Fig. 4a). SiOTIC presented redshifted absorption relative to that of SiOTC in both solution and solid films. This is believed to be associated with the different electron-withdrawing effects of the silicon atoms in the two isomers. Moreover, the SiOTIC acceptor also exhibited a higher maximum extinction coefficient with redshifted absorption relative to that of SiOTC in DCM (Fig. 4b; $1.27 \times 10^{5}$ vs. $0.99 \times 10^{5} \mathrm{M}^{-1} \mathrm{~cm}^{-1}$ ), which would benefit harvesting solar light and enhancing $J_{\mathrm{SC}}$. Both isomer films presented broader absorption ranges $(500-800 \mathrm{~nm})$ and redshifted maximum absorption peaks $(699 \mathrm{~nm}$ for SiOTC and $717 \mathrm{~nm}$ for 

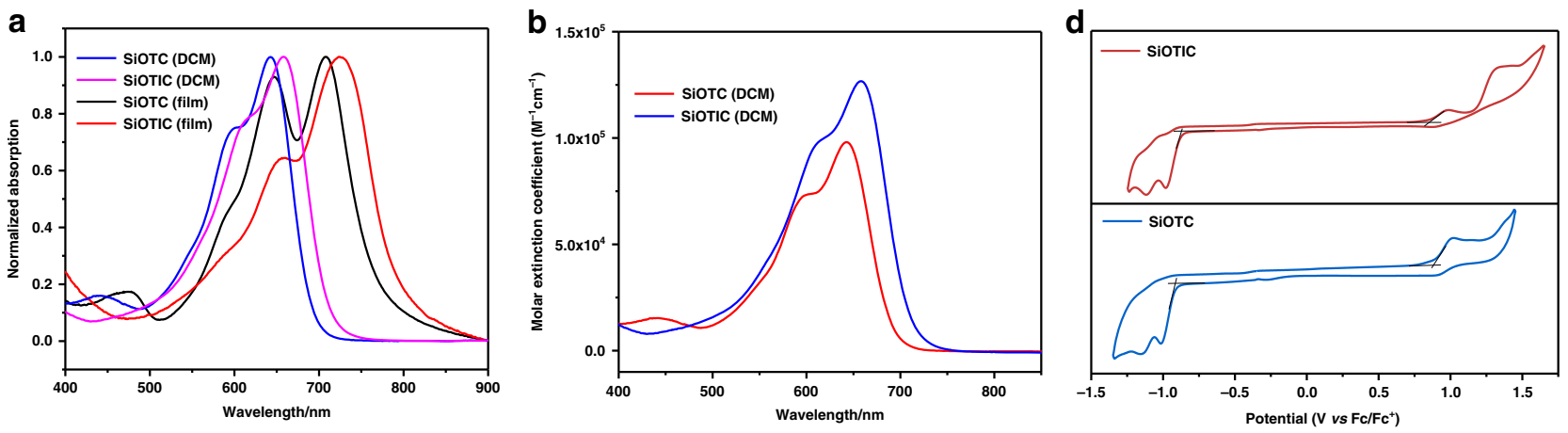

C

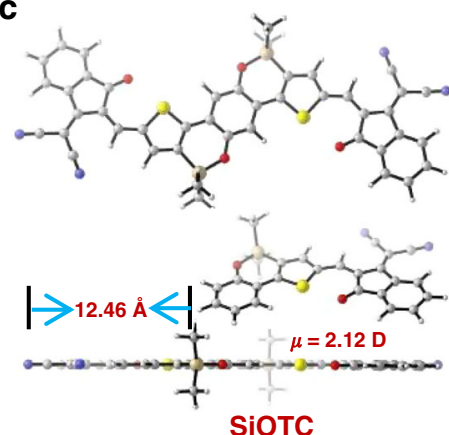

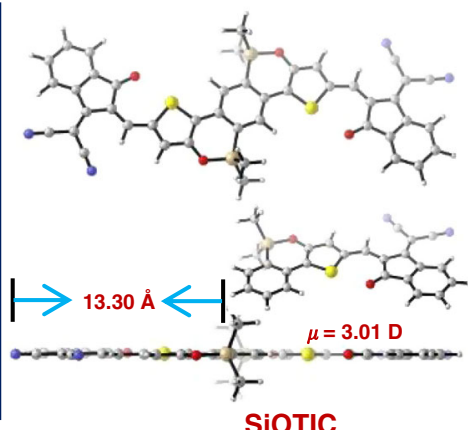

e

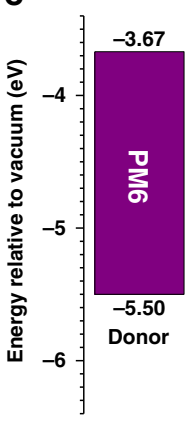

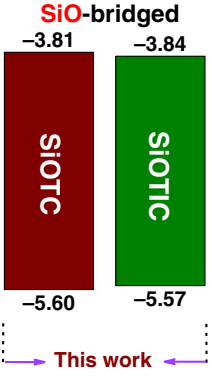

Fig. 4 Molecular physicochemical and optical properties of the acceptors. a UV-vis absorption of SiOTC and SiOTIC in DCM and film. b Comparison of the molar extinction coefficient between SiOTC and SiOTIC in DCM. c Optimized molecular geometries of SiOTC and SiOTIC by DFT calculations. d Cyclic voltammograms of SiOTC and SiOTIC in solution. e Energy level diagram of the donor and acceptor photovoltaic materials.

SiOTIC), indicating that the films of the two isomers showed stronger $\pi-\pi$ stacking interactions and more ordered aggregation. Furthermore, the $0-1$ absorption peak of the SiOTC acceptor is significantly strengthened in the solid film, indicating the stronger aggregation of SiOTC, which may lead to a complicated aggregation morphology and could be harmful to electron separation and transportation in the organic photovoltaic device. In contrast, the $0-1$ absorption peak of the SiOTIC acceptor in the film is decreased in comparison with that of its solution. We rationalized that the more compact alkyl chains of SiOTIC may prevent severe aggregation, which would benefit the solution processability in device fabrication. The optical bandgaps of the two acceptors were also estimated from the absorption edge, as shown in Table 1. The optical bandgap of the SiOTIC film is smaller than that of SiOTC (1.55 vs. $1.61 \mathrm{eV})$, which would benefit enhancing $J_{\mathrm{SC}}$ and harvesting low-energy sunlight.

DFT calculations were carried out to understand the essential differences in the backbone conformation and dipole moment of these two isomers. The side chains of the two isomers were simplified to methyl groups for the simulation. As shown in Fig. 4c, both SiOTC and SiOTIC possessed highly coplanar conformations. The main difference between SiOTC and SiOTIC is from the steric position of the alkyl groups on the silicon atom in the molecular geometries. SiOTIC showed a longer distance of 13.30 A between the center of the side chain and the edge of the IC end group than SiOTC (12.46 $\AA$ ), which allows more space for intermolecular antiparallel stacking of the terminal IC moieties. The dipole moments of the half SiOTC and SiOTIC molecules were also estimated to be $2.12 \mathrm{D}$ and $3.01 \mathrm{D}$, respectively. The larger dipole moment of the half SiOTIC molecule is responsible for the stronger intramolecular charge transfer and redshifted absorption spectra.

To further study the electronic energy level and bandgap of the two isomers, electrochemical cyclic voltammetry $(\mathrm{CV})$ was used to probe their redox properties, as shown in Fig. $4 \mathrm{~d}$. In terms of the onset reduction and oxidation potentials, the LUMO/HOMO energy levels of SiOTC and SiOTIC were estimated to be -3.81 / $-5.60 \mathrm{eV}(1.79 \mathrm{eV})$ and $-3.84 /-5.57 \mathrm{eV}(1.73 \mathrm{eV})$, the trend of which is in agreement with that obtained from DFT calculations. SiOTIC has an up-shifted HOMO compared to that of SiOTC, which could be attributed to the position effects of silicon atoms in the isomeric acceptors. As shown in Fig. 4e and Supplementary Figs. 1 and 2, the SiO-bridged acceptors SiOTC and SiOTIC possess high-lying energy levels for both the LUMO and HOMO relative to those of $\mathrm{C}$-analog IDIC ${ }^{58,59}$ and CO-analogs TPTC and TPTIC $^{65}$. The DFT calculations revealed that the better planarity and an enhanced push-pull effect between the electron donor/acceptor units on SiOTC/SiOTIC, as shown in Supplementary Figs. 3 and 4, is responsible for these changes in the energy level. This result suggested that the $\mathrm{SiO}$-bridged acceptors have the potential to deliver a higher open-circuit voltage $\left(V_{\mathrm{oc}}\right)$ in a polymer solar cell (PSC) device than those of the C- and CObridged analog-based devices. Both thermogravimetric analysis (TGA) and differential scanning calorimetry (DSC) were employed to investigate the thermal stability of these two isomers (Supplementary Fig. 5). Thermogravimetric analysis (TGA) revealed that both isomers possess fairly good thermal stability with thermal decomposition temperatures $\left(\mathrm{T}_{\mathrm{d}}\right)$ of $5 \%$ weight loss at $339^{\circ} \mathrm{C}$ for SiOTC and $323^{\circ} \mathrm{C}$ for SiOTIC. The existence of the obvious melting peak in the first heating of the DSC testing revealed that there are crystallite structures in both isomers. Notably, SiOTIC shows a higher melting temperature $\left(289.5^{\circ} \mathrm{C}\right)$ than SiOTC $\left(276.3^{\circ} \mathrm{C}\right)$, indicating that SiOTIC may undergo more compact stacking in the film, which is harder to disrupt in device fabrication; thus, thermal annealing might be needed. In general, all these studies of optical properties, electronic energy levels, and crystallinity proved that the incorporation of silicon and oxygen atoms and the isomerization of the fused-ring central 
unit played vital roles in the properties of the two $n$-type electron acceptors.

Photovoltaic performance. The strong absorption at 400-700 nm, as shown in Supplementary Fig. 6a, of the wide-bandgap donor polymer PM6 is perfectly complementary to the absorption spectra of the two SiO-bridged electron acceptors. In addition, the energy levels of PM6 $\left(E_{\mathrm{HOMO}}=-5.50 \mathrm{eV} ; E_{\mathrm{LUMO}}=-3.67 \mathrm{eV}\right.$; estimated from the CV profile of PM6, as shown in Supplementary Fig. 6b) also match well with those of the SiO-bridged acceptors (Fig. 5e). Furthermore, the photoluminescence (PL) spectra of the pure SiOTC \& SiOTIC films and the blend films with PM6 were measured and are shown in Supplementary Fig. 7. SiOTIC shows much stronger PL emission than SiOTC in the region of 730-1000 nm. Compared with the pure films, both PM6/ SiOTC and PM6/SiOTIC binary blends exhibit complete PL quenching, indicating that efficient photoinduced charge transfer occurs in the films between the donor and acceptor molecules, which is a prerequisite for achieving high photovoltaic performance. Then, we fabricated bulk heterojunction PSCs by employing PM6 as the donor and SiOTC or SiOTIC as the acceptor to assess the potential of these SiO-bridged small molecules as promising acceptors with a conventional device structure of ITO/PEDOT: PSS/PM6: acceptors/PNDIT-F3N/Ag. The effects of the regiochemistry and the SiO-synergistic effects on the photovoltaic performance of the two isomeric n-type semiconductors were systematically investigated. The donor/acceptor weight ratio in the devices was optimized to be 1:1.2 in CB solvent with $0.5 \%$ DIO additive. The tested current density-voltage $(J-V)$ and external quantum efficiency (EQE) curves of the SiOTC- and SiOTIC-based PSCs are presented in Fig. 5a, b, respectively. The extracted performance parameters are summarized in Table 2. Unfortunately, the PSCs based on as-cast PM6:SiOTC showed poor PCEs of $1.73 \%$ with very low $J_{\text {sc }}$ and FF values. Annealing treatment also did not significantly improve the performance of the SiOTC-based device (1.96\% PCE). In sharp contrast, we were pleased to find that the isomer SiOTIC-based device exhibited a decent PCE of $10.04 \%$ upon annealing treatment. The significantly increased performance of the SiOTIC-based device was attributed to the simultaneously enhanced $V_{\mathrm{oc}}(0.92 \mathrm{~V})$ and FF $(74.86 \%)$ values. From the EQE profiles of these devices, the SiOTIC-based device exhibited an elevated photoresponse from 400 to $800 \mathrm{~nm}$ with EQE beyond $60 \%$ in comparison with that of the SiOTCbased device. The different aggregation behaviors of the two isomeric siloxy-bridged acceptors in the blend active layers of the PSCs are responsible for the large difference in photovoltaic performance between the two acceptor-based devices. It is important to note that the SiOTIC-based device shows better photovoltaic performance than the reported devices using C-bridged analogs (IDIC) $^{58,59}$ and CO-bridged analogs as the acceptors ${ }^{61,63,64}$. As the absorption of SiOTIC is complementary to that of PM6 and Y6 and the LUMO/HOMO levels of SiOTIC $(-3.84 /-5.57 \mathrm{eV})$ were also between those of PM6 $(-3.67 /-5.50 \mathrm{eV})$ and Y6 $(-4.10 /-5.65 \mathrm{eV})$, as shown in Supplementary Fig. 8, SiOTIC is capable of facilitating electron transfer from PM6 to Y6 via cascade charge transfer in devices. Thus, we expanded the application of SiOTIC as the third component for ternary PSCs to realize further improvements of the classical efficient photovoltaic system in power conversion efficiency ${ }^{66}$. SiOTIC was mixed with a binary blend of PM6 and Y6 to fabricate an efficient ternary device. A PM6:Y6:SiOTIC weight ratio of 1:1.1:0.1 was employed. Owing to the simultaneous improvement in $V_{\text {oc }}$ and FF, the PCE (16.58\%) of the ternary device was considerably higher than that of the corresponding binary device based on PM6:Y6 (15.68\%). The $J-V$ and EQE curves of these binary and ternary devices are shown in 
a
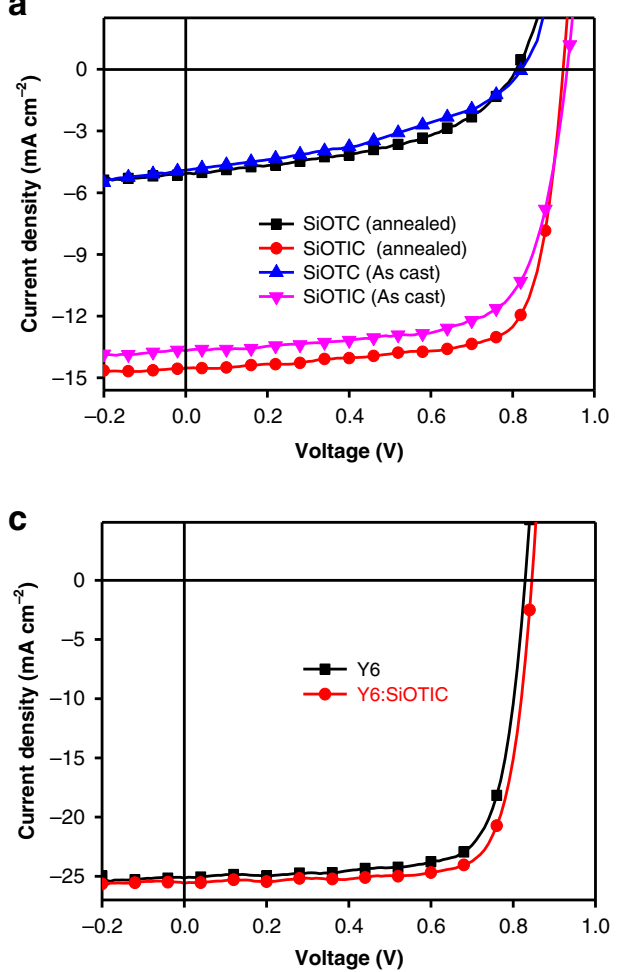

b

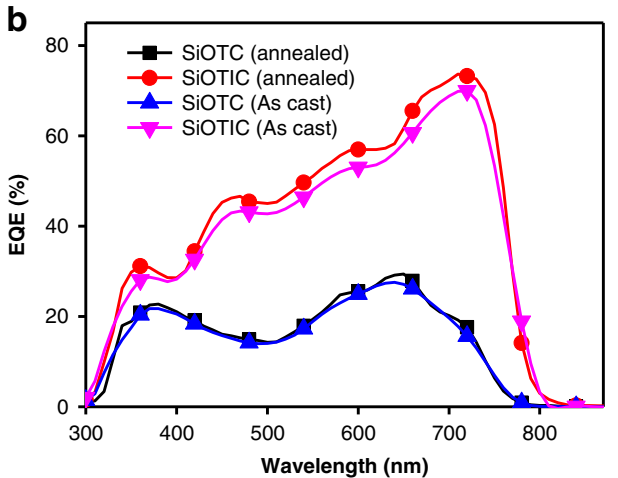

d

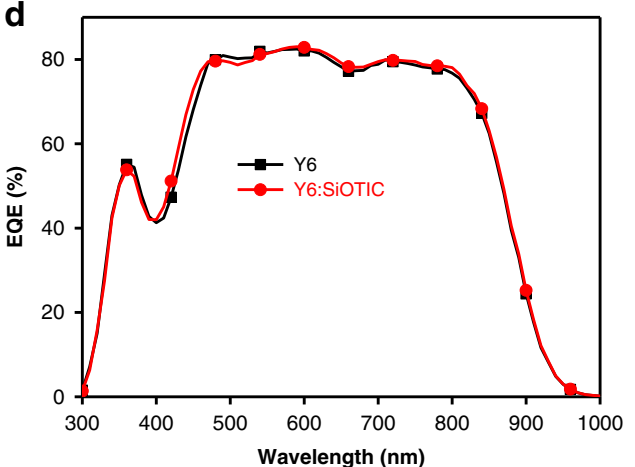

Fig. 5 Device performance of the corresponding solar cells. a J-V curves of the PM6:SiOTIC-based binary device under $100 \mathrm{~mW} \mathrm{~cm}^{-2} \mathrm{AM} 1.5 \mathrm{G}$ irradiation. b The corresponding EQE curves of the PM6:SiOTIC-based binary device. c J-V curves of the PM6:Y6 and PM6:Y6/SiOTIC-based binary and ternary devices under $100 \mathrm{~mW} \mathrm{~cm}^{-2}$ AM $1.5 \mathrm{G}$ irradiation. $\mathbf{d}$ EQE curves of the corresponding devices.

Table 2 The performance parameters of SiOTC- and SiOTIC-based PSCs with PM6 as the donor under $100 \mathrm{~mW} \mathrm{~cm}^{-2}$ AM $1.5 \mathrm{G}$ irradiation ${ }^{a}$.

\begin{tabular}{|c|c|c|c|c|c|}
\hline Acceptors & $V_{o c}[V]$ & $J_{s c}\left[\mathrm{~mA} \mathrm{~cm}{ }^{-2}\right]$ & $J_{C a l}[\mathrm{~mA} \mathrm{~cm}-2]$ & FF [\%] & PCE [\%] \\
\hline SiOTC (annealed) & $0.81(0.81 \pm 0.001)^{a}$ & $5.06(4.85 \pm 0.21)$ & 4.91 & $47.77(47.08 \pm 0.68)$ & $1.96(1.77 \pm 0.18)$ \\
\hline SiOTIC (as cast) & $0.93(0.93 \pm 0.002)$ & $13.68(13.40 \pm 0.28)$ & 13.06 & $69.44(68.16 \pm 1.26)$ & $8.86(8.40 \pm 0.44)$ \\
\hline Y6 & $0.83(0.83 \pm 0.001)$ & $25.14(24.79 \pm 0.34)$ & 24.67 & $75.15(73.86 \pm 1.28)$ & $15.68(15.27 \pm 0.40)$ \\
\hline Y6: SiOTIC (1.1:0.1) & $0.85(0.85 \pm 0.001)$ & $25.29(24.94 \pm 0.33)$ & 24.91 & $77.11(75.87 \pm 1.23)$ & $16.58(16.29 \pm 0.38)$ \\
\hline
\end{tabular}

Fig. $5 \mathrm{c}, \mathrm{d}$, and the corresponding photovoltaic parameters are also presented in Table 2. It is worth noting that the determined hole and electron mobilities of binary and ternary blends (Supplementary Fig. 9 and Supplementary Table 1) are in accordance with the different device performances. Incorporation of SiOTIC as a guest acceptor significantly enhanced the electron mobility, leading to more balanced carrier mobility in contrast to the PM6/Y6 binary blend, which is responsible for the improvement in the performance of ternary devices. These results indeed indicate that cooperation between silicon and oxygen in $\pi$-systems is a promising strategy for improving the photovoltaic properties of acceptor materials.

To understand the "isomeric effects" on charge transport and recombination in the binary blend, the hole and electron mobility and $J_{\text {sc }}$ versus illumination were also measured, as shown in Supplementary Figs. 10 and 11. The mobility calculated by means of the SCLC method is shown in Table S2. The hole mobility of the blend film was $8.5 \times 10^{-6}$ and $1.7 \times 10^{-4} \mathrm{~cm}^{2} \mathrm{~V}^{-1} \mathrm{~s}^{-1}$ for SiOTC and SiOTIC, respectively. Furthermore, the electron mobility of the blend film was $5.3 \times 10^{-6}$ and $1.3 \times 10^{-4} \mathrm{~cm}^{2} \mathrm{~V}^{-1} \mathrm{~s}^{-1}$ for
SiOTC and SiOTIC, respectively. The extremely low electron and hole mobility of the blend film with SiOTC indicates that a loose molecular conformation of SiOTC might lead to complicated stacking and diversified aggregation modes, which are harmful to carrier transport. In contrast, the blend film with SiOTIC had much higher electron and hole mobility, and the balanced hole and electron transport of SiOTIC was also better than that of the other isomer-based blend films, implying that a more compact alkyl chain is of great benefit to suppression of the over-stacking, results in more ordered aggregation and ensures the carrier transfer. The results were also supported by the carrier recombination of the devices, and the slopes from Supplementary Fig. 11 for SiOTC and SiOTIC were 0.90 and 0.97 , respectively. To further study the effect of structure variation on device performance, transient photovoltage (TPV) and transient photocurrent (TPC) measurements were conducted to investigate the carrier lifetime and sweeping out time, as shown in Supplementary Fig. 12 and Supplementary Table 3. The carrier lifetime $(\tau)$ was determined by fitting to be 0.60 and $0.74 \mu$ for SiOTC- and SiOTIC-based devices, respectively. 

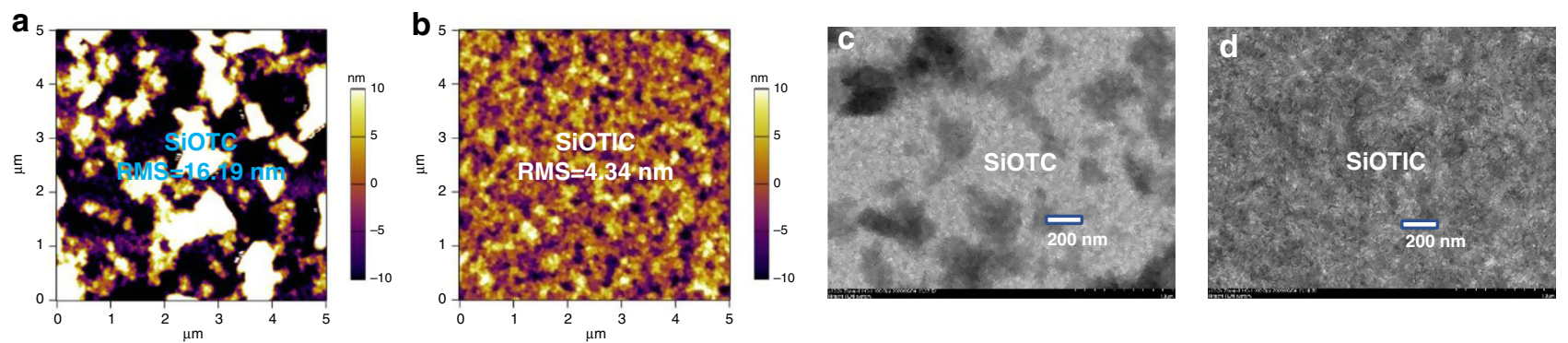

Fig. 6 AFM and TEM images of the PM6:SiOTC and PM6:SiOTIC blend films. a AFM images $(5 \mu \mathrm{m} \times 15 \mu \mathrm{m})$ of the PM6: SiOTC blend film. b AFM images $(5 \mu \mathrm{m} \times 15 \mu \mathrm{m})$ of the PM6:SiOTIC blend film. c TEM image of the PM6: SiOTC blend film. d TEM image of the PM6:SiOTIC blend films.
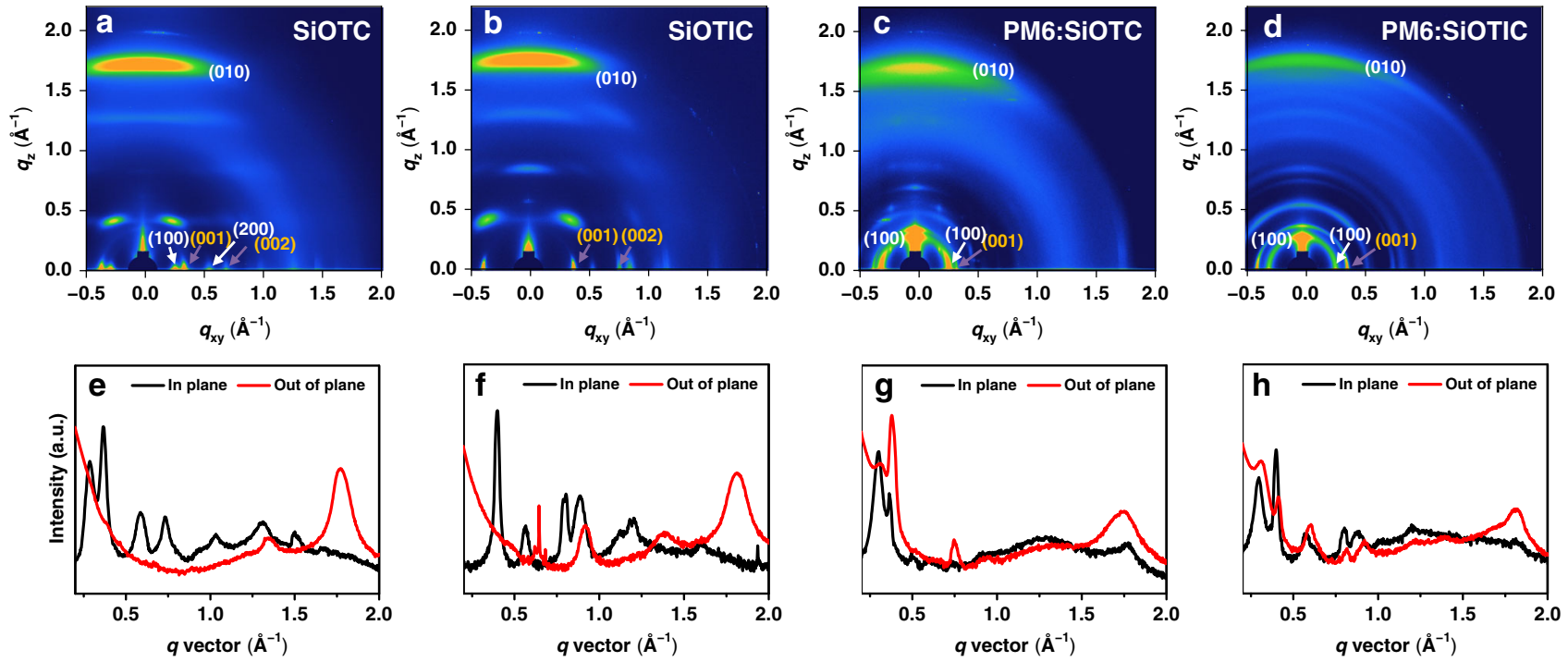

Fig. 7 GIWAXS results. a GIWAXS pattern of the SiOTC film. b GIWAXS pattern of the SiOTIC film. c GIWAXS pattern of the PM6:SiOTC blend film. d GIWAXS pattern of the PM6:SiOTIC film. e The line-cuts of in-plane and out-of-plane SiOTC. $\mathbf{f}$ The line-cuts of in-plane and out-of-plane SiOTIC. $\mathbf{g}$ Line cuts of the in-plane and out-of-plane PM6:SiOTC blend films. h Line cuts of the in-plane and out-of-plane PM6:SiOTIC blend films.

The longer lifetime indicated less recombination, which is consistent with the above description. The sweeping out time $\left(t_{s}\right)$ was calculated from the ternary device curves as 0.44 and $0.37 \mu$ s for SiOTC- and SiOTIC-based devices, respectively. The faster $t_{s}$ for the SiOTIC-based device exhibited more efficient charge extraction around electrodes, consistent with the higher $J_{\mathrm{sc}}$ and FF values for the device.

Morphological characterization. To study the influences of phase-separated morphology on the performance of devices, atomic force microscopy (AFM) and transmission electron microscopy (TEM) were conducted to study the film surface of the blend film with these two acceptors. As shown in Fig. 6a, b (AFM), the SiOTC-based film displayed a very rough surface with a root-mean square roughness (RMS) value of $16.19 \mathrm{~nm}$. In contrast, the SiOTIC film had a smooth surface morphology with an RMS value of $4.34 \mathrm{~nm}$. The excessive aggregation that appeared in the SiOTC-based film led to a large domain size that hindered charge separation. From the TEM images (Fig. 6c, d), the film with SiOTC had obvious clusters among the fibers, and the SiOTIC film had a dispersive fibrillar nanostructure, which is beneficial for producing efficient exciton separation. These results showed that fine modulation of the molecular structure was crucial for the desired morphology in the solid-state and influenced the optimized performance of PSCs.
Grazing-incidence wide-angle X-ray scattering (GIWAXS) measurements were also carried out to investigate the molecular packing and crystallinity in the neat and blend films. The twodimensional (2D) GIWAXS patterns are shown in Fig. 7a-d, and the intensity profiles in the out-of-plane (OOP) and in-plane (IP) directions are plotted in Fig. 7e-h. The peak positions, d-spacing, and coherence lengths (CLs) are also presented in Supplementary Table 4. Both SiOTC and SiOTIC in neat films exhibit obvious $\pi$ $-\pi(010)$ stacking peaks in the OOP direction at $\mathrm{q}_{\mathrm{z}}=1.774 \AA^{-1}$ (SiOTC) and $1.813 \AA^{-1}$ (SiOTIC), indicating that such molecules are highly ordered with preferential "face-on" orientation. Shorter OOP $\pi-\pi$ stacking distances $\left(d_{\pi}=3.466 \AA\right.$ in SiOTIC \& $d_{\pi}=$ $3.541 \AA$ in SiOTC) were observed in SiOTIC, implying that SiOTIC possesses more compact $\pi-\pi$ stacking, which is beneficial to intermolecular electron transport in films. However, the pure SiOTC film exhibits higher crystallinity than SiOTIC with a dominant face-on orientation, as reflected by the longer CLs of the OOP (010) peaks (69.158 $\AA$ in SiOTC and $58.714 \AA$ in SiOTIC). Notably, both SiOTC and SiOTIC display (001) reflections at $0.366 \AA^{-1}$ (SiOTC) and $0.398 \AA^{-1}$ (SiOTIC) in the IP direction, originating from backbone ordering, which would facilitate intermolecular electron transport.

According to the scattering profiles of blend films, the PM6/ SiOTIC blend film presented closer $\pi-\pi$ stacking $\left(\mathrm{q}_{\mathrm{z}}=1.815 \AA^{-1}\right.$, $\mathrm{d}=3.461 \AA$ in the PM6/SiOTIC blend; $\mathrm{q}_{\mathrm{z}}=1.760 \AA^{-1}, \mathrm{~d}=3.569$ $\AA$ in the PM6/SiOTC blend), higher crystalline characteristics 
(CLs: $70.139 \AA$ in the PM6/SiOTIC blend and $53.058 \AA$ in the PM6/SiOTC blend) in the OOP direction and stronger IP backbone (001) stacking $\left(\mathrm{q}_{\mathrm{xy}}=0.398 \AA^{-1}, \mathrm{~d}=15.777 \AA\right.$ and $\mathrm{CL}=279.564 \AA$ in the PM6/SiOTIC blend; $\mathrm{q}_{\mathrm{xy}}=0.365 \AA^{-1}$, $\mathrm{d}=17.234 \AA$ and $\mathrm{CL}=223.717 \AA$ ) in the PM6/SiOTC blend than in the PM6/SiOTIC blend, which facilitates charge transport in the vertical direction and suppresses charge carrier recombination, as evidenced by the improved fill factor, charge mobility and $J_{s c}$ in the SiOTIC-based device.

\section{Discussion}

In summary, two isomeric SiO-bridged ladder-type $\pi$-skeletons (SiO5Ts) were designed and synthesized for the first time by using two different tin-free synthetic pathways, showing the advantage of fewer synthetic steps over conventional aromatic fused-rings. In addition, compared with their CO-bridged analogs, the SiO-bridged ladder-type skeletons possess enhanced backbone planarity and lower-lying energy levels of both the LUMO and HOMO. To explore its potential application in organic electronics, two $n$-type A-D-A structured organic semiconductors were constructed by the use of the two isomeric skeletons as the central fused ring D-units. The isomeric effects and the silicon and oxygen synergistic effects of the two SiObridged acceptors on the physicochemical and photovoltaic performance were systematically investigated. The results revealed that the molecular geometries, photophysical properties, and aggregation behaviors of the acceptors can be finely adjusted via alteration of the silicon atom positions on the fused-ring central units, thus resulting in a significant effect on the blend film morphology, charge mobility, and photovoltaic performance. As a result, the PSCs based on SiOTIC delivered a decent PCE of up to $10.04 \%$, while that of the SiOTC-based device only reached $1.96 \%$ PCE. Higher device performance can be expected with further efforts on device engineering, such as using appropriate donors, interfacial interlayers, and morphology control. The promising PCE of up to $10.04 \%$ given by our first few trials is higher than those of the 5-membered IDT and 6-membered CO5T analogbased PSCs reported in the literature, unambiguously confirming the considerable potential of the SiO-bridged ladder-type $\pi$ skeletons for further development of new high-performance semiconductor materials. The structure-property relationship is beneficial to the discovery of new n-type organic semiconductor acceptors for high-performance PSCs.

Reporting summary. Further information on research design is available in the Nature Research Reporting Summary linked to this article.

\section{Data availability}

The authors declare that the data supporting the findings of this study are available within the article and its Supplementary Information Files as well as from the corresponding authors on request.

Received: 12 July 2020; Accepted: 22 October 2020;

Published online: 16 November 2020

\section{References}

1. Huitema, H. E. A. et al. Plastic transistors in active-matrix displays. Nature 414, 599 (2001).

2. Forrest, S. R. \& Thompson, M. E. Introduction: organic electronics and optoelectronics. Chem. Rev. 107, 923-925 (2007).

3. Root, S. E., Savagatrup, S., Printz, A. D., Rodriquez, D. \& Lipomi, D. J. Mechanical properties of organic semiconductors for stretchable, highly flexible, and mechanically robust electronics. Chem. Rev. 117, 6467-6499 (2017).
4. Arias, A. C., MacKenzie, J. D., McCulloch, I., Rivnay, J. \& Salleo, A. Materials and applications for large area electronics: solution-based approaches. Chem. Rev. 110, 3-24 (2010)

5. Ostroverkhova, O. Organic optoelectronic materials: mechanisms and applications. Chem. Rev. 116, 13279-13412 (2016).

6. Rogers, J. A., Someya, T. \& Huang, Y. G. Materials and mechanics for stretchable electronics. Science 327, 1603-1607 (2010).

7. Gelinck, G., Heremans, P., Nomoto, K. \& Anthopoulos, T. D. Organic transistors in optical displays and microelectronic applications. Adv. Mater. 22, 3778-3798 (2010).

8. Sirringhaus, H. 25th Anniversary article: organic field-effect transistors: the path beyond amorphous silicon. Adv. Mater. 26, 1319-1335 (2014).

9. Paterson, A. F. et al. Recent progress in high-mobility organic transistors: a reality check. Adv. Mater. 30, 1801079 (2018).

10. Kelley, T. W. et al. Recent progress in organic electronics: materials, devices, and processes. Chem. Mater. 16, 4413-4422 (2004).

11. Geffroy, B., le Roy, P. \& Prat, C. Organic light-emitting diode (OLED) technology: materials, devices and display technologies. Polym. Int. 55, 572-582 (2006).

12. Wang, C., Dong, H., Hu, W., Liu, Y. \& Zhu, D. Semiconducting $\pi$-conjugated systems in field-effect transistors: a material odyssey of organic electronics. Chem. Rev. 112, 2208-2267 (2012).

13. Facchetti, A. $\pi$-Conjugated polymers for organic electronics and photovoltaic cell applications. Chem. Mater. 23, 733-758 (2011).

14. Mei, J., Diao, Y., Appleton, A. L., Fang, L. \& Bao, Z. Integrated materials design of organic semiconductors for field effect transistors. J. Am. Chem. Soc. 135, 6724-6746 (2013).

15. Hains, A. W., Liang, Z., Woodhouse, M. A. \& Gregg, B. A. Molecular semiconductors in organic photovoltaic cells. Chem. Rev. 110, 6689-6735 (2010).

16. Wu, J.-S., Cheng, S.-W., Cheng, Y.-J. \& Hsu, C.-S. Donor-acceptor conjugated polymers based on multifused ladder-type arenes for organic solar cells. Chem. Soc. Rev. 44, 1113-1154 (2015)

17. Lee, J., Kalin, A. J., Yuan, T., Al-Hashimic, M. \& Fang, L. Fully conjugated ladder polymers. Chem. Sci. 8, 2503-2521 (2017).

18. Chen, J., Yang, K., Zhou, X. \& Guo, X. Ladder-type heteroarene-based organic semiconductors. Chem. Asian J. 13, 2587-2600 (2018).

19. Cai, Z., Awais, M. A., Zhang, N. \& Yu, L. Exploration of syntheses and functions of higher ladder-type $\pi$-conjugated heteroacenes. Chemistry 4 , 2538-2570 (2018).

20. Yamaguchi, S., Xu, C. \& Okamoto, T. Ladder $\pi$-conjugated materials with main group elements. Pure Appl. Chem. 78, 721-730 (2006).

21. Fukazawa, A. \& Yamaguchi, S. Ladder $\pi$-conjugated materials containing main-group elements. Chem. Asian J. 4, 1386-1400 (2009).

22. He, X. \& Baumgartner, T. Conjugated main-group polymers for optoelectronics. RSC Adv. 3, 11334-11350 (2013).

23. Yang, X., Zhou, G. \& Wong, W.-Y. Functionalization of phosphorescent emitters and their host materials by main-group elements for phosphorescent organic light-emitting devices. Chem. Soc. Rev. 44, 8484-8575 (2015).

24. Parke, S. M., Boone, M. P. \& Rivard, E. Marriage of heavy main group elements with $\pi$-conjugated materials for optoelectronic applications. Chem. Commun. 52, 9485-9505 (2016).

25. Ponomarenko, S. A. \& Kirchmeyer, S. Conjugated organosilicon materials for organic electronics and photonics. Adv. Polym. Sci. 235, 33-110 (2011).

26. Xu, C., Wakamiya, A. \& Yamaguchi, S. Ladder oligo(p-phenylenevinylene)s with silicon and carbon bridges. J. Am. Chem. Soc. 127, 1638-1639 (2005).

27. Yamaguchi, S., Xu, C. \& Tamao, K. Bis-silicon-bridged stilbene homologues synthesized by new intramolecular reductive double cyclization. J. Am. Chem. Soc. 125, 13662-13663 (2003).

28. Bin, H. et al. 11.4\% Efficiency non-fullerene polymer solar cells with trialkylsilyl substituted 2D-conjugated polymer as donor. Nat. Commun. 7, 13651 (2016).

29. Bin, H. et al. Effect of alkylsilyl side-chain structure on photovoltaic properties of conjugated polymer donors. Adv. Energy Mater. 8, 1702324 (2018).

30. Zhang, Z. \& Zhu, X. Bis-silicon-bridged stilbene: A core for small-molecule electron acceptor for high-performance organic solar cells. Chem. Mater. 30, 587-591 (2018).

31. Ashraf, R. S. et al. Silaindacenodithiophene semiconducting polymers for efficient solar cells and high-mobility ambipolar transistors. Chem. Mater. 23, 768-770 (2011).

32. Coughlin, J. E., Henson, Z. B., Welch, G. C. \& Bazan, G. C. Design and synthesis of molecular donors for solution-processed high-efficiency organic solar cells. Acc. Chem. Res. 47, 257-270 (2014).

33. Chen, J. \& Cao, Y. Silole-containing polymers: chemistry and optoelectronic properties. Macromol. Rapid Commun. 28, 1714-1742 (2007).

34. Wong, W. W. H., Hooper, J. F. \& Holmes, A. B. Silicon analogues of polyfluorene as materials for organic electronics. Aust. J. Chem. 62, 393-401 (2009). 
35. Ohshita, J. Conjugated oligomers and polymers containing dithienosilole units. Macromol. Chem. Phys. 210, 1360-1370 (2009).

36. Kushida, Y., Nagano, T. \& Hanaoka, K. Silicon-substituted xanthene dyes and their applications in bioimaging. Analyst 140, 685-695 (2015).

37. Wang, J.-Y. et al. Benzobis(silolothiophene)-based low bandgap polymers for efficient polymer solar cells. Chem. Mater. 23, 765-767 (2011).

38. Ni, W. et al. Dithienosilole-based small-molecule organic solar cells with an efficiency over 8\%: investigation of the relationship between the molecular structure and photovoltaic performance. Chem. Mater. 27, 6077-6084 (2015).

39. Yuan, M., Yang, P., Durban, M. M. \& Luscombe, C. K. Low bandgap polymers based on silafluorene containing multifused heptacylic arenes for photovoltaic applications. Macromolecules 45, 5934-5940 (2012).

40. Nian, Y. et al. Silaindacenodithiophene-based fused-ring non-fullerene electron acceptor for efficient polymer solar cells. Chin. J. Chem. 36, 495-501 (2018).

41. Love, J. A. et al. Silaindacenodithiophene-based molecular donor: morphological features and use in the fabrication of compositionally tolerant, high-efficiency bulk heterojunction solar cells. J. Am. Chem. Soc. 136, 3597-3606 (2014).

42. Hou, J., Chen, H.-Y., Zhang, S., Li, G. \& Yang, Y. Synthesis, characterization, and photovoltaic properties of a low band gap polymer based on silolecontaining polythiophenes and 2,1,3-benzothiadiazole. J. Am. Chem. Soc. 130, 16144-16145 (2008).

43. Xiao, Z. et al. Carbon-oxygen-bridged ladder-type building blocks for highly efficient nonfullerene acceptors. Adv. Mater. 31, 1804790 (2019).

44. Huang, C. \& Gevorgyan, V. TBDPS and Br-TBDPS protecting groups as efficient aryl group donors in Pd-catalyzed arylation of phenols and anilines. $J$. Am. Chem. Soc. 131, 10844-10845 (2009).

45. Shintani, R., Maciver, E. E., Tamakuni, F. \& Hayashi, T. Rhodium-catalyzed asymmetric synthesis of silicon-stereogenic dibenzooxasilines via enantioselective transmetalation. J. Am. Chem. Soc. 134, 16955-16958 (2012).

46. Zhao, W.-T., Lu, Z.-Q., Zheng, H., Xue, X.-S. \& Zhao, D. Rhodium-catalyzed 2-arylphenol-derived Six-membered silacyclization: straightforward access toward dibenzooxasilines and silicon-containing planar chiral metallocenes. ACS Catal. 8, 7997-8005 (2018).

47. Brook, A. G. Molecular rearrangements of organosilicon compounds. Acc. Chem. Res. 7, 77-84 (1974).

48. Wadsworth, A. et al. Critical review of the molecular design progress in nonfullerene electron acceptors towards commercially viable organic solar cells. Chem. Soc. Rev. 48, 1596-1625 (2019).

49. Zhang, G. et al. Nonfullerene acceptor molecules for bulk heterojunction organic solar cells. Chem. Rev. 118, 3447-3507 (2018).

50. Hou, J., Inganas, O., Friend, R. H. \& Gao, F. Organic solar cells based on nonfullerene acceptors. Nat. Mater. 17, 119-128 (2018).

51. Cheng, P., Li, G., Zhan, X. \& Yang, Y. Next-generation organic photovoltaics based on non-fullerene acceptors. Nat. Photon 12, 131-142 (2018).

52. Yan, C. et al. Non-fullerene acceptors for organic solar cells. Nat. Rev. Mater. 3, 18003 (2018).

53. Cui, C. Recent progress in fused-ring based nonfullerene acceptors for polymer solar cells. Front. Chem. 6, 404 (2018).

54. Jiang, Z.-Q., Wang, T.-T., Wu, F.-P., Lin, J.-D. \& Liao, L.-S. Recent advances in electron acceptors with ladder type backbone for organic solar cells. $J$. Mater. Chem. A. 6, 17256-17287 (2018).

55. He, D., Zhao, F., Jiang, L. \& Wang, C. A-D-A small molecule acceptors with ladder-type arenes for organic solar cells. J. Mater. Chem. A. 6, 8839-8854 (2018).

56. Acceptor-donor-acceptor type molecules for high performance organic photovoltaics - chemistry and mechanism. Chem. Soc. Rev. 49, 2828-2842 (2020).

57. Lin, Y. et al. An electron acceptor challenging fullerenes for efficient polymer solar cells. Adv. Mater. 27, 1170-1174 (2015).

58. Lin, Y. et al. A facile planar fused-ring electron acceptor for as-cast polymer solar cells with $8.71 \%$ efficiency. J. Am. Chem. Soc. 138, 2973-2976 (2016).

59. Lin, Y. et al. Structure evolution of oligomer fused-ring electron acceptors toward high efficiency of as-cast polymer solar cells. Adv. Energy Mater. 6, 1600854 (2016).

60. Li, X. et al. Simplified synthetic routes for low cost and high photovoltaic performance n-type organic semiconductor acceptors. Nat. Commun. 10, 519 (2019).

61. Xiao, Z. et al. A carbon-oxygen-bridged ladder-type building block for efficient donor and acceptor materials used in organic solar cells. Sci. Bull. 62, 1331-1336 (2017).
62. Yang, L. et al. Molecular consideration for small molecular acceptors based on ladder-type dipyran: influences of O-functionalization and $\pi$-bridges. $A d v$. Funct. Mater. 28, 1705927 (2018).

63. Zhou, Y. et al. High efficiency small molecular acceptors based on novel Ofunctionalized ladder-type dipyran building block. Nano Energy 45, 10-20 (2018).

64. Wen, S. et al. Pyran-bridged indacenodithiophene as a building block for constructing efficient A-D-A-type nonfullerene acceptors for polymer solar cells. ChemSusChem 11, 360-366 (2018).

65. Li, X. et al. Effects of fused-ring regiochemistry on the properties and photovoltaic performance of n-type organic semiconductor acceptors. $J$. Mater. Chem. A. 6, 15933-15941 (2018).

66. Yuan, J. et al. Single-junction organic solar cell with over $15 \%$ efficiency using fused-ring acceptor with electron-deficient core. Joule 3, 1140-1151 (2019).

\section{Acknowledgments}

D.Z. is grateful for the financial support from the National Natural Science Foundation of China (22022103, 22071114, 21871146), the National Key Research and Development Program of China (2019YFA0210500), the 1000-Talent Youth Program (020/BF180181), the Natural Science Foundation of Tianjin (18JCYBJC20400), and the Fundamental Research Funds for the Central Universities and Nankai University. F.H. is grateful for support from the Shenzhen Nobel Prize Scientists Laboratory Project (C17213101), Guangdong Innovative and Entrepreneurial Research Team Program (2016ZT06G587) and Shenzhen Sci-Tech Fund (KYTDPT20181011104007). We thank the SUSTech Core Research Facilities for the AFM and TEM measurements. Z.-G.Z. is grateful for the financial support from the National Natural Science Foundation of China (51722308).

\section{Author contributions}

Y.Q. and Y.Z. (Yue Zhou) performed chemical synthesis and property characterization of these SiOT5s. H.C. and J.Y. designed and fabricated PSC devices. Y.Z. (Yulin Zhu) conducted the AFM and TEM experiments. Y.C. and C.Y. performed the GIWAXS measurements. C.-W.J. and B.Q. carried out the DFT calculation. D.Z., F.H., Z.-G.Z., and Y.L. developed the concept, designed the experiments, supervised the project and wrote the manuscript.

\section{Competing interests}

The authors declare no competing interests.

\section{Additional information}

Supplementary information is available for this paper at https://doi.org/10.1038/s41467 020-19605-z.

Correspondence and requests for materials should be addressed to Z.-G.Z., F.H. or D.Z

Peer review information Nature Communications thanks the anonymous reviewers for their contribution to the peer review of this work. Peer reviewer reports are available.

Reprints and permission information is available at http://www.nature.com/reprints

Publisher's note Springer Nature remains neutral with regard to jurisdictional claims in published maps and institutional affiliations.

\section{(i)}

Open Access This article is licensed under a Creative Commons Attribution 4.0 International License, which permits use, sharing, adaptation, distribution and reproduction in any medium or format, as long as you give appropriate credit to the original author(s) and the source, provide a link to the Creative Commons license, and indicate if changes were made. The images or other third party material in this article are included in the article's Creative Commons license, unless indicated otherwise in a credit line to the material. If material is not included in the article's Creative Commons license and your intended use is not permitted by statutory regulation or exceeds the permitted use, you will need to obtain permission directly from the copyright holder. To view a copy of this license, visit http://creativecommons.org/ licenses/by/4.0/.

() The Author(s) 2020 